\title{
Burayidi, Michael; Allen, Adriana; Twigg, John; Wamsler, Christine (Hrsg.) (2020): The Routledge Handbook of Urban Resilience.
}

\author{
Sonja Deppisch \\ Eingegangen: 23. November 2020 - Angenommen: 7. Dezember 2020 - Online veröffentlicht: 2. Februar 2021
}

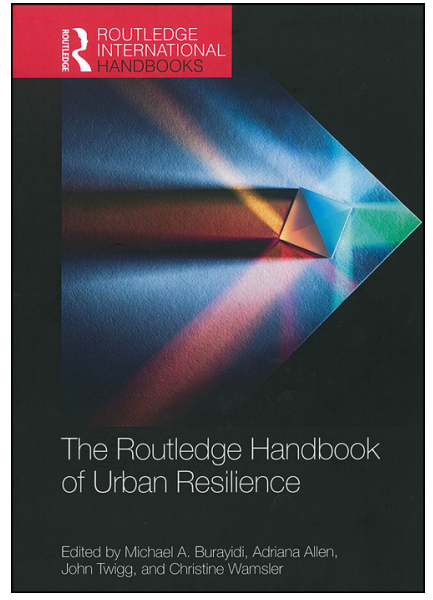

Im vorliegenden Handbuch werden unterschiedliche disziplinäre Perspektiven auf urbane Resilienz vereint. Dabei handelt es sich um eine Sammlung von einzelnen Beiträgen zu urbaner Resilienz, die kritische, eher bekannte und weithin vertretene Positionen und Themen mit entsprechenden Fallstudien vertritt, aber auch, wenngleich zu einem geringeren Anteil, selteneren Perspektiven einen Raum gibt.

Teil I ist betitelt mit „Critical review from different disciplinary perspectives" und gibt einen Einblick in verschiedene Aspekte der (eher theoretischen) Resilienzdiskussion bezogen auf den städtischen Kontext. Der Kürze der einzelnen Kapitel geschuldet, geht das nicht ins argumentative

Dr. Sonja Deppisch, HafenCity Universität Hamburg, Henning-Voscherau-Platz 1, 20457 Hamburg sonja.deppisch@hcu-hamburg.de

(c) (1) (2) ( 2021 Deppisch; licensee oekom verlag. This Open Access article is published under the Creative Commons Attribution-ShareAlike 4.0 International Licence.
Detail, doch vermittelt es den Lesenden, insbesondere denen, die nicht mit dem Resilienzdiskurs vertraut sind, einen guten Einblick in die verschiedenen Diskussionen. Beispiele hierfür sind der Unterschied von Resilienz zum Konzept der Nachhaltigkeit oder die Unterscheidung in allgemeine und spezifische Resilienz sowie das Ungenügen von Resilienzkonzeptionen für sozialökologische Systeme, die sich in den Naturwissenschaften verorten, wenn diese angewendet werden auf sozial komplexe städtische Kontexte. Ein neuer Aspekt taucht auf durch die Verbindung von Risikoreduzierung und Resilienzaufbau mit mindfulness, vor allem in Bezug auf katastropheninduzierte Risiken, wobei hier Resilienzaufbau weitgehend mit Risikominimierung gleichgesetzt wird. Insgesamt betrachtet differiert das Abstraktionsniveau der unterschiedlichen Beiträge in diesem ersten Teil beträchtlich.

Der zweite Teil trägt den Titel „Urban systems under stress“. Hier liegt in einigen Kapiteln teilweise der Fokus auf Vulnerabilität und nicht in allen wird ein konzeptioneller Bogen zurück zur Resilienz geschlagen. Vulnerabilität ist als Thema in einem Handbuch zu urbaner Resilienz nicht unpassend, allerdings hätte es hier einer stärkeren editorischen Einordnung oder zumindest Hinleitung in konzeptioneller Hinsicht bedurft. Einige Kapitel stellen diesen Bezug in der zweiten Hälfte beziehungsweise am Ende allerdings auch her. Als Stressoren wird im zweiten Teil eine Vielfalt aufgezeigt unter anderem von den Auswirkungen von Klimawandel auf kritische städtische Infrastrukturen über Hochwasserereignisse bis hin zur Schrumpfung von Städten. Auch gewähren die Beiträge Einblicke in nicht nur thematisch, sondern auch geographisch und soziokulturell unterschiedliche Kontexte urbaner Resilienz, mit mehreren Beiträgen zu den USA, zu Mexico, China und den Philippinen. 
Im dritten Teil „Dimensions of resilience“ wird zunächst nicht ganz klar, was damit eigentlich gemeint ist, da dies weder in einem verbindenden Klammertext noch in der Einleitung zum Handbuch erläutert wird. So fehlt auch der konkrete Zusammenhang zwischen den einzelnen Kapiteln, die von einem Literatur-Review zur Messung von Resilienz im urbanen Kontext über Fallstudien mit eher losem Resilienzbezug bis zur empirischen Analyse urbaner Resilienz ganz unterschiedliche Beiträge aufweisen. Nach der Lektüre sämtlicher Beiträge des dritten Teils wird klarer, was mit den Dimensionen von Resilienz gemeint ist: Resilienz durch grüne Infrastruktur, Resilienz (grauer) kritischer Infrastrukturen, kulturelle und soziale Resilienz, sozialökologische Resilienz, ökonomische sowie Katastrophenresilienz, auch mit einem langfristigeren Bezug beim Klimawandel. Für ein Handbuch ist es ausdrücklich schade, dass dies nicht explizit gemacht wird; so unterscheiden sich die Kapitel stark in ihrer Charakteristik und erscheinen als neben- beziehungsweise nacheinandergestellt. Auch wird bei manchen Kapiteln nicht Resilienz als das eigentliche Thema fokussiert. $\mathrm{Zu}$ diesem Eindruck trägt auch bei, dass in manchen Kapiteln explizit definiert wird, was unter Resilienz verstanden wird, während in anderen Beiträgen eine explizite theoretisch-konzeptionelle Ausführung fehlt.

Der vierte und letzte Teil des Buches mit dem Titel „Resilience building in practice“ vereint zehn Beiträge, darunter acht Fallstudien aus verschiedenen Kontinenten und zwei konzeptionelle Beiträge. Während sich die Fallstudien beispielsweise aus Malawi, Sierra Leone, Chile, Nepal und Kolumbien hauptsächlich auf den Umgang mit Naturkatastrophen in Städten beziehen, gehen Fallstudien in Seoul (Südkorea) sowie Europa und Australien auf Dachgärten und naturbasierte Lösungen ein. Zwei Kapitel leisten eher generelle beziehungsweise konzeptionelle Auseinandersetzungen ohne expliziten Fallstudienbezug. Dabei behandelt Kapitel 28 („Understanding the fabric of large urban areas to improve disaster planning and recovery") die besondere
Situation des Katastrophenmanagements in großräumigen städtischen Gebieten und wie dort soziale und physikalische Resilienz aufgebaut werden kann. Im letzten Kapitel des Buches werden die Ergebnisse der einzelnen Buchteile noch einmal zusammengefasst und anhand der bekannten W-Fragen zu Resilienz sortiert: Resilienz von was, wo und wann, Resilienz für wen und von wem. Dieses letzte Kapitel ist hilfreich, um einen Überblick über relevante Fragen und Ergebnisse zu gewinnen, die in den Buchteilen und einzelnen Kapiteln angesprochen werden. Zum Schluss gibt es also noch eine Art roten Faden, der allerdings nicht ganz kongruent zum Buchaufbau ist und auch nicht alle einzelnen Kapitel des gesamten Buches durchwebt.

Insgesamt trifft der Sammelband nicht alle Erwartungen, die mit einem Handbuch verbunden werden, sondern es handelt sich um eine lesenswerte Sammlung von Perspektiven und Fallstudien, die dem Thema der Resilienz und der urbanen Resilienz gewidmet sind, mit einem Schwerpunkt auf Katastrophenresilienz. Nicht immer wird klar, worin genau der Unterschied zwischen Risikovermeidung und Resilienz liegt. Die Perspektive der engineering resilience wird in manchen Beiträgen kritisiert und als unzureichend für das Erreichen tatsächlicher urbaner Resilienz angesehen. Hier hätte eine Einordnung und editorische Arbeit seitens der Herausgeber/-innen die Leserschaft gerade eines Handbuches stärker an die Hand nehmen können. Insgesamt fehlt der rote Faden für ein Handbuch, der die Beiträge in ihrer Provenienz und Stoßrichtung strukturell oder hinleitend einordnet, insbesondere für diejenigen, die mit der Diskussion um urbane Resilienz weniger vertraut sind.

\section{Vollständige bibliographische Angaben des rezensierten Werkes: \\ Burayidi, Michael; Allen, Adriana; Twigg, John; Wamsler, Christine (Hrsg.) (2020): The Routledge Handbook of Urban Resilience. London/New York: Routledge. 54 Ta- bellen, 76 Abbildungen, 502 Seiten.}

